\title{
Stellar and GCR Production of Lithium in the Milky Way
}

\author{
F. Matteucci \\ Department of Astronomy, University of Trieste, Via G.B. Tiepolo, 11, \\ I-34131 Trieste, Italy \\ D. Romano
}

SISSA/ISAS, Via Beirut, 2-4, I-34014 Trieste, Italy

\begin{abstract}
Lithium production from several stellar sources (C-stars, massive AGB stars, Type II supernovae and novae) as well as from galactic cosmic rays (GCR) is included in a succesfull model for the chemical evolution of the Milky Way in order to predict the evolution of the ${ }^{7} \mathrm{Li}$ abundance $\left(\mathrm{A}(\mathrm{Li})=\log \left({ }^{7} \mathrm{Li} / \mathrm{H}\right)+12\right)$ as a function of $[\mathrm{Fe} / \mathrm{H}]$. From comparison with the oservational data we infer the following conclusions: 1) $\mathrm{Li}$ production from novae seems to be necessary to explain the steep rise of the $\mathrm{Li}$ abundance for metallicities larger than $[\mathrm{Fe} / \mathrm{H}]=-1.0 \mathrm{dex}, 2)$ $\mathrm{Li}$ production from SNe II should be less than assumed before in order to reproduce the long plateau observed for $\mathrm{A}(\mathrm{Li})$ for low metallicities, 3) $\mathrm{Li}$ production from C-stars is negligible relative to $\mathrm{Li}$ production from massive AGB stars which is instead a necessary Li- source, 4) Li production from GCR should contribute by no more than $20 \%$ to the solar $\mathrm{Li}$ abundance.
\end{abstract}

\section{Introduction}

The observed $\mathrm{A}(\mathrm{Li})$ vs. $[\mathrm{Fe} / \mathrm{H}]$ in dwarf stars shows that the $\mathrm{Li}$ abundance in Pop I and T Tauri stars is at least a factor of ten higher than in Pop II stars.

This fact can be interpreted in different ways:

- either the primordial Li abundance is high (that observed in Pop I stars) implying that this element is progressively destroyed inside stars and that non-standard Big Bang nucleosynthesis is required,

- or the primordial $\mathrm{Li}$ abundance is low and coincides with that measured in Pop II stars which show a remarkable uniformity of $\mathrm{Li}$ abundance (the so-called Spite plateau). In such a case, beyond the fact that the estimated primordial $\mathrm{Li}$ abundance is in agreement with the predictions of the standard Big Bang nucleosynthesis, an efficient production of Li during the Galaxy lifetime is required to explain the $\mathrm{Li}$ abundance in Pop I stars.

In the following we will adopt this point of view.

A recent data compilation by Romano et al. (1999) concerning lithium abundances in disk and halo dwarfs with $T_{\text {eff }} \geq 5700 \mathrm{~K}$ has shown: 
- a well defined plateau extending perhaps up to $[\mathrm{Fe} / \mathrm{H}] \geq-0.5 \mathrm{dex}$,

- a general trend of an increasing $\mathrm{Li}$ abundance with increasing $[\mathrm{Fe} / \mathrm{H}](>$ - $1.5 \mathrm{dex}$ ) reaching quite high $\mathrm{Li}$ abundances in the T Tauri stars and in the local interstellar medium (ISM).

These observational data (Figure 1) together with the fact that the ${ }^{7} \mathrm{Li} /{ }^{6} \mathrm{Li}$ ratio in the solar system is $\sim 12$ as opposed to the value of this ratio expected from the GCR production $(\sim 2)$, suggest that a substantial fraction of the ${ }^{7} \mathrm{Li}$ in the Galaxy should have a stellar origin (see Reeves 1993). The data also show a large spread in the Li abundance of disk stars whereas the spread observed in the plateau is much smaller. The smaller spread in Pop II stars can be probably explained as an effect of metallicity. D'Antona and Mazzitelli (1984) suggested that main-sequence Li depletion is inhibited in low metallicity stars.

On the other hand, main-sequence Li destruction occurs in high metallicity stars because of deeper surface convection due to the higher opacity of Pop I stars. Therefore, the spread in Pop I stars is mainly due to age effects, the older stars being the most Li depleted. Because of this, models of galactic chemical evolution predicting the $\mathrm{Li}$ abundance in the ISM should fit the upper envelope of the $\mathrm{A}(\mathrm{Li})$ vs. $[\mathrm{Fe} / \mathrm{H}]$ data.

In this paper, we adopt a succesfull model of chemical evolution of the Milky Way reproducing the majority of observational constraints (Chiappini et al. 1997, 1999 where a detailed description can be found), and we use it to compute the evolution of the $\mathrm{Li}$ abundance in the interstellar medium (ISM) by taking into account all the possible stellar Li-producers as well as the Li produced by spallation of GCR. The results are then compared with the observational data in order to put constraints on the origin of lithium.

\section{Stellar Li-sources}

\subsection{Observations of Li-rich stars}

Observations of massive AGB and M supergiants in the Magellanic Clouds indicate the presence of a large $\mathrm{Li}$ abundance together with enhanced s-process elements, from which one infers that they are in their thermal pulsing (TP) phase, but they are not C-stars (Smith and Lambert 1989, 1990; Plez et al. 1993).

On the other hand, Galactic C-stars with luminosities from $M_{b o l} \simeq-6$ to - 3.5 show in some cases very high $\mathrm{Li}$ abundances (Abia et al. $1991 ; 1993$ ) but the total amount of mass that they can loose is negligible to have an effect on Galactic Li production.

Therefore, there is a clear observational indication that low and intermediate mass stars $\left(1 \leq M / M_{\odot} \leq 8\right)$ can be Li-producers, especially the more massive ones (see D'Antona and Matteucci 1991).

Concerning the other possible Li producers such as Type II SNe and novae, there are no observational indications about $\mathrm{Li}$ production. As a consequence, the nucleosynthesis prescriptions for these objects are only theoretical. 


\subsection{Nucleosynthesis prescriptions}

\section{C-stars $\left(2-5 M_{\odot}\right)$}

$\mathrm{Li}$ is produced during thermal pulses and then destroyed: survival may last a few thermal pulses but the actual mass of produced and ejected $\mathrm{Li}$ is quite uncertain. We assume the same prescriptions adopted by D'Antona and Matteucci (1991) $(\mathrm{A}(\mathrm{Li})=3.85$ dex per star $)$.

\section{High mass AGB stars}

Sackmann and Boothroyd (1992) showed that stars of masses 4-6 $M_{\odot}$, in the luminosity range $-6 \leq M_{b o l} \leq-7$, can reach $\mathrm{A}(\mathrm{Li})=4.5$ dex in the envelope as a consequence of hot bottom burning. We assumed that each star produces either $\mathrm{A}(\mathrm{Li})=3.5$ or $4.15 \mathrm{dex}$, in agreement with Matteucci et al. (1995) and in agreement with the observations.

\section{Type II SNe}

$\nu$-induced nucleosynthesis can create a substantial amount of Li. The flux of neutrinos following the core collapse to form a neutron star becomes so great that it can induce substantial transmutation. $\mu$ and $\tau$ neutrinos excite heavy elements and $\mathrm{He}$ to particle unbound levels. The evaporation of a neutron or proton and the back reaction of these nucleons on other species alters the outcome of traditional nucleosynthesis calculations. ${ }^{7} \mathrm{Li}$ is partly produced in the H-shell but mostly in the He- and Si-shells (Woosley et al. 1990).

\section{Novae.}

Very recent nucleosynthesis calculations (José and Hernanz 1998) suggest that the explosive formation of ${ }^{7} \mathrm{Li}$ in novae can produce an average $\mathrm{Li}$ mass per nova of $\left\langle M_{L i}\right\rangle=1.8-7.5 \times 10^{-7} M_{\odot}$. The important aspect of novae is that they start restoring Li into the ISM only after a timescale of $>1 \mathrm{Gyr}$, due to the slow evolution leading to the nova outburst (white dwarfs in binary systems). Details on the nova evolution relative to the Galactic enrichment in lithium can be found in Romano et al. (1999).

Table 1. Input parameters and results.

\begin{tabular}{ccccccc}
\hline & C-stars & M-AGB & SNeII & novae & $\mathrm{A}(\mathrm{Li})_{\mathrm{SS}}$ & $\mathrm{A}(\mathrm{Li})_{\mathrm{ISM}}$ \\
\hline $\mathrm{A}$ & 3.85 & 4.15 & WW95 & no & 2.91 & 2.90 \\
$\mathrm{~B}$ & 3.85 & 4.15 & WW95 & yes & 3.12 & 3.24 \\
$\mathrm{C}$ & no & 3.50 & WW95/2 & yes & 2.95 & 3.12 \\
$\mathrm{C}+$ GCRs & no & 3.50 & WW95/2 & yes & 3.21 & 3.39 \\
\hline \hline
\end{tabular}

\section{Model Results}

We have computed the evolution in time of the $\mathrm{Li}$ abundance by changing the nucleosynthesis prescriptions. Table 1 summarizes the input parameters of the different models and some results. In particular the first column of Table 1 indicates the model, the second the prescriptions adopted for C-stars with the assumed amount of Li produced by each star expressed in terms of $\mathrm{A}(\mathrm{Li})$, the same is for the massive AGB stars in column 3. Column 4 indicates the sources of the adopted prescriptions for type II SNe: in a couple of models the produced 


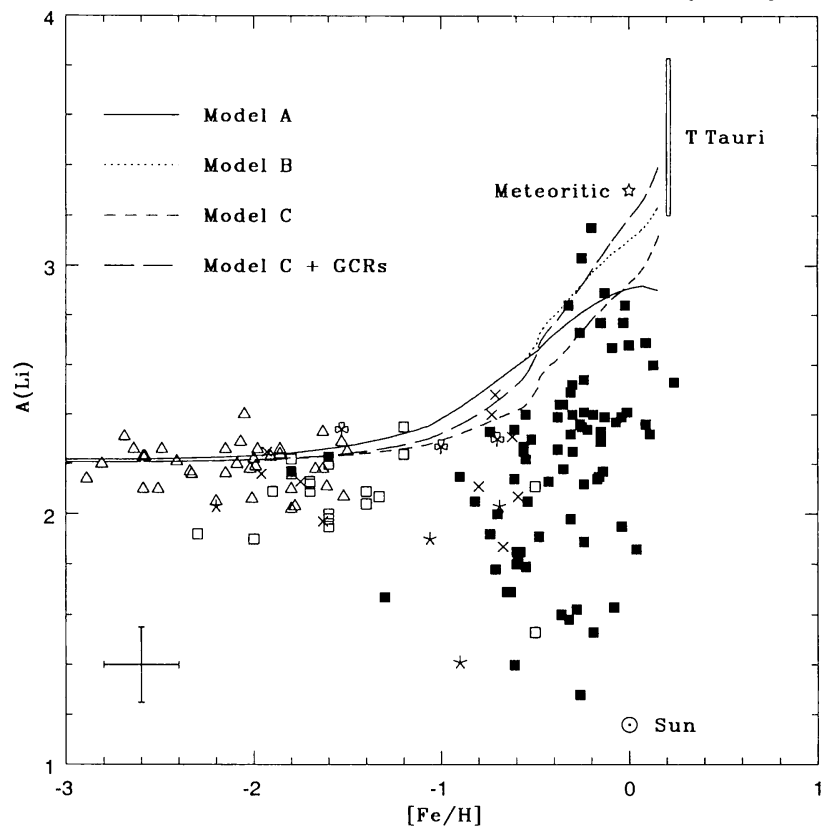

Figure 1. Observed and predicted $\mathrm{A}(\mathrm{Li})$ versus $[\mathrm{Fe} / \mathrm{H}]$ in the solar neighbourhood. Data are from the compilation of Romano et al. 1999. The abundances of $\mathrm{Li}$ in the $\mathrm{T}$ Tauri are indicated as well well as the $\mathrm{Li}$ abundance in the sun. Models are labelled A, B, C, C+GCR according to the text.

lithium has been reduced to one half. Column 5 indicates if we have adopted the novae prescriptions or not (the Li mass produced by novae is the one mentioned in the previous paragraph). Finally in column 6 and 7 we report the predicted $\mathrm{Li}$ abundance for the ISM at the time of the formation of the solar system and for the local ISM, respectively. Concerning the production of ${ }^{7} \mathrm{Li}$ from $\mathrm{GCR}$ we assumed the results of Lemoine et al. (1998) and we computed only one model including all the Li prescriptions (stellar plus GCR).

In Figure 1 we show a comparison between the predictions of the models of Table 1 and the observational data. As mentioned before, a good model of chemical evolution should reproduce the upper envelope of these data ignoring the observed spread which indicates the progressive Li destruction inside stars and does not reflect the $\mathrm{Li}$ abundance of the ISM. An inspection of Figure 1 indicates that the models including a late Li production from novae are necessary to explain the steep increase of the $\mathrm{Li}$ abundance for $[\mathrm{Fe} / \mathrm{H}]>-1.0 \operatorname{dex}$ (Models $\mathrm{B}, \mathrm{C}, \mathrm{C}+\mathrm{GCR})$. Among these models the one which also includes a late production of $\mathrm{Li}$ by GCR seems to be the best although the amount of Li contributed by GCR to the solar system $\mathrm{Li}$ abundance should not exceed $10-20 \%$ of the total. In particular the model presented here (Model C+GCR) contributes for more than $20 \%$ of the solar lithium. Therefore a model intermediate between Model B (no GCR production) and Model C+GCR would produce the best fit to the data. Another consideration arising from the inspection of Figure 1 is that the neutrino spallation process acting in SNe II seems to overproduce Li at 
low metallicities and that reducing this quantity by at least one half produces a better agreement with the data.

\section{Conclusions}

In this paper we have presented some predictions for the evolution of the abundance of ${ }^{7} \mathrm{Li}$ in the ISM of the Milky Way. We have assumed that the $\mathrm{Li}$ abundance observed in the local ISM as well as in young stars as T Tauri is the result of the stellar lithium production. We have envisaged several possible Li producers and suggested that novae or alternatively a stellar $\mathrm{Li}$ source acting at late times in the Galactic evolutionary history ( $\mathrm{t}>1 \mathrm{Gyr}$ ) are necessary to explain the observational data. Other sources like massive AGB stars are also necessary to produce the solar system and present time observed $\mathrm{Li}$ abundance. Lithium production from GCR is not fundamental but it can help if is acting at late times and does not produce more than $10-20 \%$ of the total solar lithium as indicated by the meteoritic ${ }^{7} \mathrm{Li} /{ }^{6} \mathrm{Li}$ ratio as compared with what is expected from GCR Li production.

\section{References}

Abia, C., Boffin, H.M.J., Isern, J., \& Rebolo, R. 1991, A\&A, 245, L1

Abia, C., Isern, J., \& Canal, R. 1993, A\&A, 275, 96

Chiappini, C., Matteucci, F., \& Gratton, R. 1997, ApJ, 477, 765

Chiappini, C., Matteucci, F., Beers, T.C., \& Nomoto, K. 1999, ApJ, 515, 226

D'Antona, F., \& Matteucci, F. 1991, A\&A, 247, L37

D'Antona, F., \& Mazzitelli, I. 1984, A\&A, 138, 431

José, J., \& Hernanz, M. 1998, ApJ, 494, 680

Lemoine, M., Vangioni-Flam, E., \& Cassé, M. 1998, ApJ, 499, 735

Matteucci, F., D'Antona, F., \& Timmes, F.X. 1995, A\&A, 303, 460

Plez, B., Smith, V.V., \& Lambert, D.L. 1993, ApJ, 418, 812

Reeves, H. 1993, A\&A, 269, 166

Romano, D., Matteucci, F., Molaro, P., \& Bonifacio, P. 1999, A\&A, 352, 117

Sackmann, I.J., \& Boothroyd, A.I. 1992, ApJ, 392, L71

Smith, V.V., \& Lambert, D.L. 1989, ApJ, 345, L75

Smith, V.V., \& Lambert, D.L. 1990, ApJ, 361, L69

Woosley, S.E., Hartmann, D.H., Hoffman, R.D., \& Haxton, W.C. 1990, ApJ, 356,272 